\title{
A Rigiflex, Spontaneously Wettable Polymeric Mold for Forming Reversibly Bonded Nanocapillaries
}

\author{
Pilnam Kim and Kahp Y. Suh
}

School of Mechanical and Aerospace Engineering, Seoul National University, Seoul, 151-742, Korea

*Corresponding author: Phone: +82-2-880-9103; Fax:+82-2-880-1725; E-mail: sky4u@snu.ac.kr

This supplemental information consists of EOS plot and diagram of zeta-potential of PEG-DA film mold and some examples for cohesion failure without additional UV exposure.

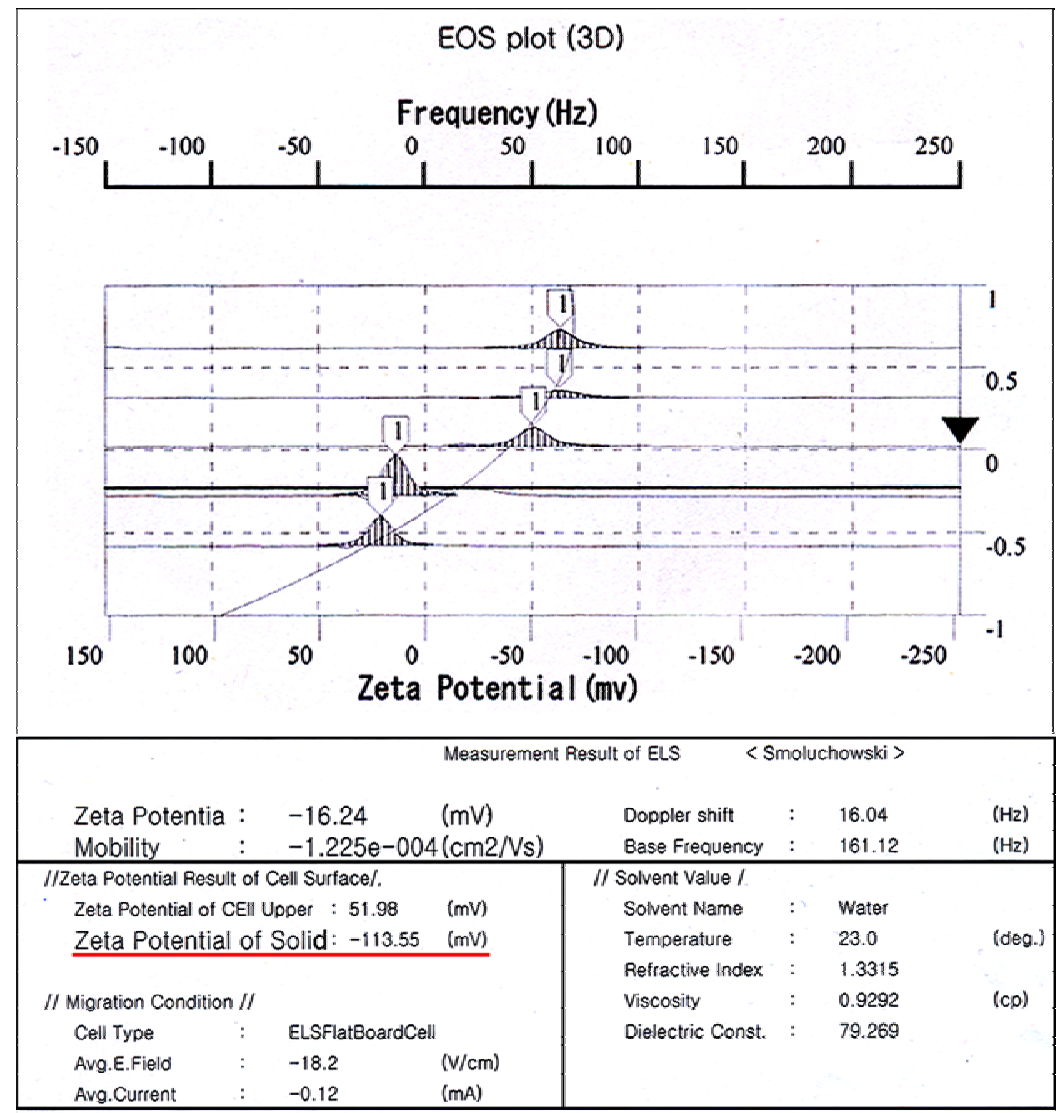

Supplemental Figure 1. EOS plot and diagram of zeta-potential of PEG-DA film mold measured by ELS (Electrophoretic Light Scattering Spectrophotometer). Highly negative surface charges (-113.55 
$\mathrm{mV}$ ) were observed on the surface of PEG-DA film mold, resulting in electrostatic attraction with gold or silicon substrate.

\section{(a) Cohesion failure of PEG-DA mold}
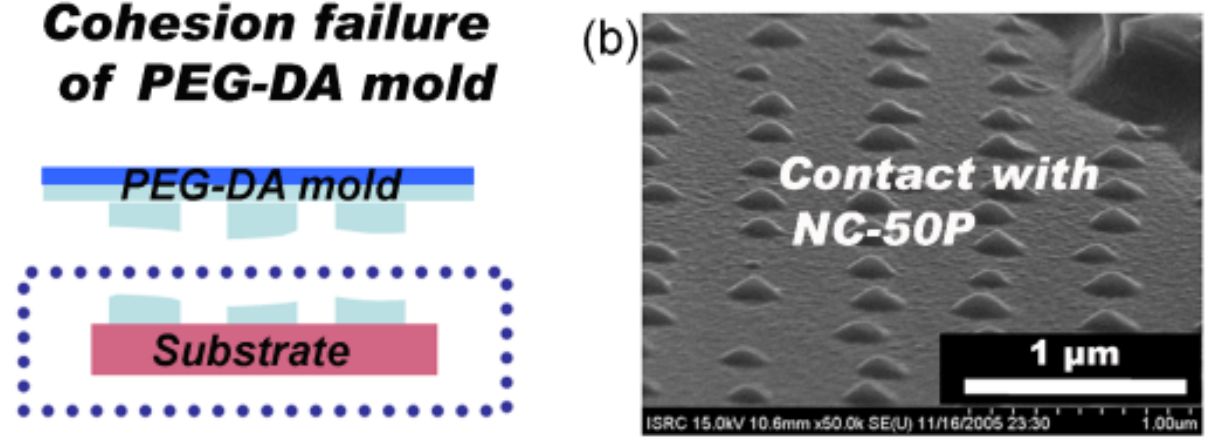

(c)
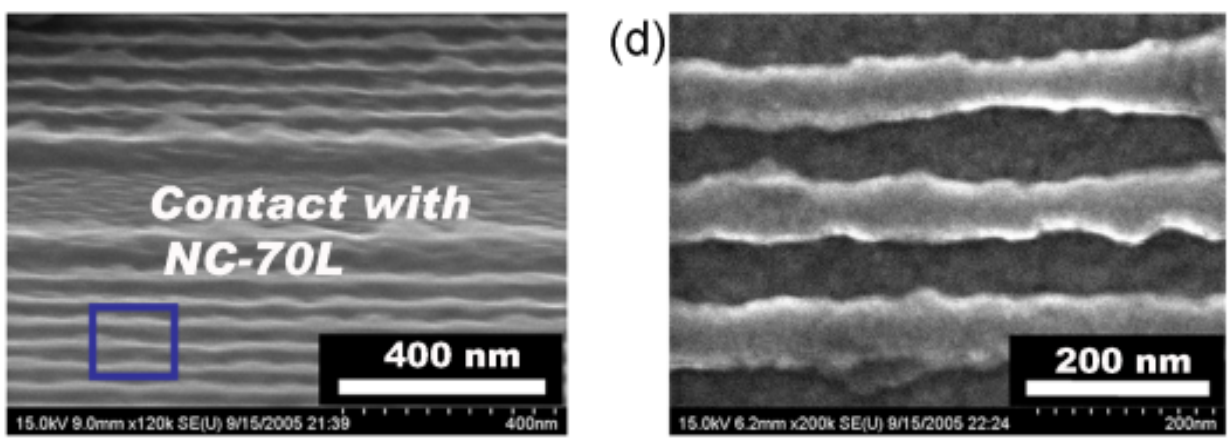

Supplemental Figure 2. (a) A schematic diagram and examples (b-d) of cohesion mechanical failure by insufficient UV-exposure time due to low cohesion strength/elastic modulus of mold. (b) An image showing transferred dots from the incompletely cured 50nm pillar mold. (c) An image showing transferred nanolines from the incompletely cured 70nm line mold. (d) A magnified image of (c). 